\title{
Shedding light on the optical properties of spider silk fiber
}

\author{
Desmond M. Chow ${ }^{1, *}$, Kenny Hey Tow ${ }^{1}$, Fritz Vollrath ${ }^{2}$, Isabelle Dicaire ${ }^{3}$, Tom Gheysens ${ }^{3}$ and Luc \\ Thévenaz ${ }^{1}$
}
${ }^{1}$ EPFL Swiss Federal Institute of Technology, Group for Fibre Optics, SCI-STI-LT, Station 11, CH-1015 Lausanne, Switzerland; ${ }^{2}$ University of Oxford, Department of Zoology, Oxford OX1 3PS, UK;
${ }^{3}$ Advanced Concepts Team, European Space Agency (ESA), Noordwijk, The Netherlands.
*desmond.chow@epfl.ch

\begin{abstract}
Optical characterisation of a Nephila edulis spider dragline silk is performed. The silk fiber transmits light up to $1400 \mathrm{~nm}$ with a propagation loss of $\sim 9 \mathrm{~dB} / \mathrm{cm}$ and birefringence of $8 \times 10^{-3}$ measured at $1302 \mathrm{~nm}$.
\end{abstract}

Keywords-Biophotonics; spider silk; optical fiber.

\section{INTRODUCTION}

In nature, spiders use their silk to weave capture webs, flying kites, prey snares and more. The potential of spider silk has spurred intense research on the many attractive properties of spider silk, prompting discussions of novel applications in different fields. For instance, the silks of spiders and silkworms are showing promise as a good biocompatible material for e.g. the enhancement of skin regeneration and nerve guides [1]. And the often exceptional material strengths can be tapped in the development of specialist textiles and composites e.g. for drones and airplanes [2]. Previously, Shao et al. showed that spider silk shrinks in water and in a range of polar solvents [3] and very recently, light guiding in spider dragline silk of Nephila clavipes has been demonstrated [4], paving the way for using silks as fiber-optic biosensors. Therefore, additional optical characterizations such as spectral transmission, propagation loss and birefringence need to be carried out on spider silk to explore its potentialities.

\section{DRAGLINE SILK}

Silks are protein threads consisting of repeating motives of polypeptides that contain both discrete crystalline and non-crystalline domains oriented along the fiber axis. In this study, a single strand of silk of the major ampullate gland controllably reeled from a female Nephila edulis was used because: (1) Nephila edulis is a well-studied species. (2) It produces thick and strong silk fibers, which facilitates easy handling. (3) The silk can be collected under controlled conditions such as reeling speed, humidity and temperature all of which affect its material properties.

Dragline silk is transparent in the vicinity of visible wavelength region and has a RI of $\sim 1.55$ [5]. Light can be guided inside when it is surrounded by air $(n=1)$. Due to the high RI contrast between silk and its surrounding (air), silk fiber exhibits multimode guidance. The scanning electron microscope (SEM) image of a silk fiber sample, with a core diameter of $5.6 \mu \mathrm{m}$, is shown in Fig. 1. The reeling process, performed at Oxford Silk group under controlled speed, resulted in a uniformly spun fiber with a smooth surface, circular cross-section and homogenous material properties.

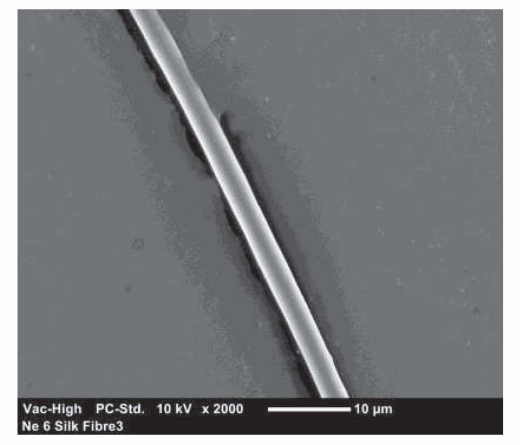

Fig. 1. SEM image of a spider silk with core diameter of $5.6 \mu \mathrm{m}$

\section{LIGHT INJECTION}

Light can be launched into the silk fiber by either evanescent field coupling or by end-fire light injection. Evanescent field coupling is performed by launching light into a half-tapered fiber with the tapered end close to that of the dragline silk. When the tapered silica fiber and the silk fiber are placed close to each other, power exchanges alternatingly between them along interaction length through evanescent field. Evanescent field coupling is a non-invasive method and more suitable for studying dragline silk in its pristine condition. However, the efficiency of evanescent field coupling depends on various factors such as wavelength, RI of silk, diameter of silk, etc. The limited bandwidth of evanescent field coupling method also prevents the study of spectral transmission. End-fire coupling method is achieved by launching light directly into the silk fiber from the fiber's end facet. A strand of silk fiber $(25 \mathrm{~mm})$ with properly prepared end surfaces is immobilized on a fixed support. Single-mode fibers attached on translation stages are used to couple light in and out the silk fiber. 


\section{OPTICAL CHARACTERIZATION}

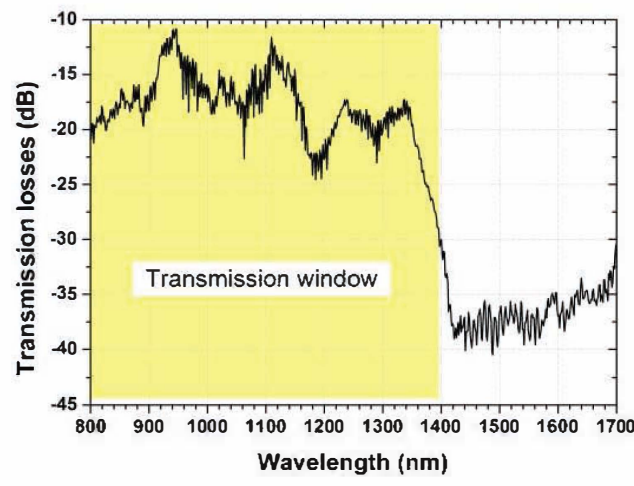

Fig. 2. Transmission loss spectrum of the silk fiber.

Spectral transmission characterization was performed by launching supercontinuum light $(800 \mathrm{~nm}-1700 \mathrm{~nm})$ into the silk fiber. An optical spectrum analyser (OSA) was used to detect the transmitted light. The transmission loss spectrum, as shown in Fig. 2, was obtained by normalizing output from input spectrum. One can notice that the transmission drops sharply beyond $1360 \mathrm{~nm}$. The wavelength range with lesser propagation loss is $900-1100 \mathrm{~nm}$. The relative transmission loss (compared with transmission loss at $940 \mathrm{~nm}$ ) in $\mathrm{C}$ band is large $(>25 \mathrm{~dB})$, therefore it is impracticable to work in this region. The $1300 \mathrm{~nm}$ wavelength region appears to be a good transmission window as the relative transmission loss is still low $(<10 \mathrm{~dB})$ enough for transmission and optical components are more available in $\mathrm{O}$ band than at $900 \mathrm{~nm}$.

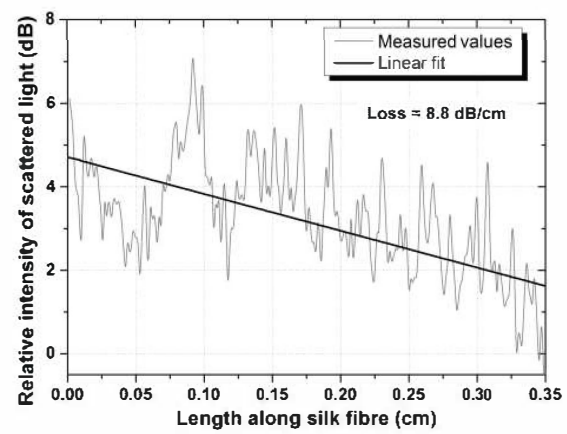

Fig. 3. Relative intensity of scattered light against longitudinal position.

Distributed scattering loss technique was used to measure the propagation loss of silk fiber due to the difficulty linked to cleaving the silk fibers. It measures the intensity of scattered light from a fiber as a function of position, with the assumption that the scattered light is homogeneous and proportional to the actual intensity of light guided in the fiber. The intensity variation of the scattered light is obtained by taking the peak intensity at each point along the propagation direction. Fig. 3 represents the relative scattered power as a function of position along the silk fiber at $1302 \mathrm{~nm}$. The measured points are linearly fitted and the corresponding slope gives the value of propagation loss $\alpha$. The propagation loss is estimated to be $9 \pm 2 \mathrm{~dB} / \mathrm{cm}$ in the $\mathrm{O}$ band.

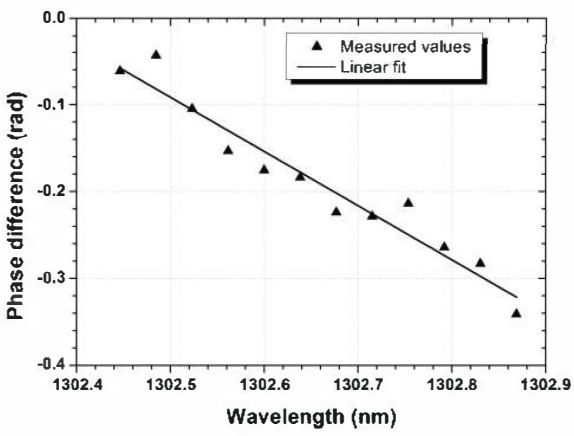

Fig. 4. Rotation angle $\varphi$ of trasmitted light against wavelength.

In order to measure the birefringence of the silk fiber, a method based on wavelength scanning [6] was used. Light from a tunable laser is launched inside the silk fiber and the transmitted light is analysed with a polarization analyser. Detuning the wavelength of the input light induces an angular rotation of the output polarization on the Poincaré sphere. Fig. 4 shows this rotation angle $\varphi$ against wavelength. From this graph, a birefringence of $8 \times 10^{-3}$ is calculated at $1302 \mathrm{~nm}$.

\section{CONCLUSION}

Light coupling inside a spider dragline silk has allowed us performing an optical characterisation of the latter: (i) transmission window extending up to $1400 \mathrm{~nm}$, (ii) a propagation loss of $9 \mathrm{~dB} / \mathrm{cm}$ and (iii) a birefringence of $8 \times 10^{-3}$ at $1302 \mathrm{~nm}$. The results show huge potential of silk fiber to be deployed as biochemical sensor, bio-compatible optical components, etc.

\section{ACKNOWLEDGMENT}

This work was done within the framework of the Ariadna study $n^{\circ}$ 14-6401 (The Silky Way) supported by the European Space Agency. The Oxford Silk group thanks ERC (SP2-GA-2008-233409) and AFOSR (FA9550-12-1-0294) for funding.

\section{REFERENCES}

1 Wendt, H., Hillmer, A., Reimers, K., Kuhbier, J.W., Schäfer-Nolte, F. Allmeling, C., Kasper, C., and Vogt, P.M.: 'Artificial skin-culturing of different skin cell lines for generating an artificial skin substitute on crossweaved spider silk fibres', PloS one, 2011, 6, (7), pp. e21833

2 Vollrath, F., and Knight, D.P.: 'Liquid crystalline spinning of spider silk', Nature, 2001, 410, (6828), pp. 541-548

3 Shao, Z., and Vollrath, F.: 'The effect of solvents on the contraction and mechanical properties of spider silk', Polymer, 1999, 40, (7), pp. 17991806

4 Huby, N., Vié, V., Renault, A., Beaufils, S., Lefevre, T., Paquet-Mercier, F., Pézolet, M., and Bêche, B.: 'Native spider silk as a biological optical fiber', Appl. Phys. Lett., 2013, 102, (12), pp. 123702

5 Little, D.J., and Kane, D.: 'Image contrast immersion method for measuring refractive index applied to spider silks', Opt. Express, 2011, 19, (20), pp. 19182-19189

6 Kikuchi, K., and Okoshi, T.: 'Wavelength-sweeping technique for measuring the beat length of linearly birefringent optical fibers', Opt. Lett., $1983,8,(2)$, pp. $122-123$ 